\title{
Checklist Supervisor
}

National Cancer Institute

\section{Source}

National Cancer Institute. Checklist Supervisor. NCI Thesaurus. Code C51810.

A person who is responsible for directing personnel involved with the coordination and completion of forms that contain a list of information regarding procedure or content validation. 